\title{
Concave iteration semigroups of linear set-valued functions
}

\author{
by Jolanta Olko (Kraków)
}

\begin{abstract}
We consider a concave iteration semigroup of linear continuous set-valued functions defined on a closed convex cone in a separable Banach space. We prove that such an iteration semigroup has a selection which is also an iteration semigroup of linear continuous functions. Moreover it is majorized by an "exponential" family of linear continuous set-valued functions.
\end{abstract}

Let $X$ be a real normed space. We denote by $n(X)$ the family of all nonempty subsets of $X$. The families $c(X)$ and $c c(X)$ consist of all compact and all compact convex members of $n(X)$, respectively. We consider the space $c c(X)$ with the Hausdorff metric $h$ induced by the norm in $X$. For the properties of the Hausdorff metric and the convergence in the space $(c c(X), h)$ see [1], [2] or [3]. Some of them needed here are also collected in $[5]$.

If $X, Y, Z$ are nonempty sets and $F: X \rightarrow n(Y)$ is any set-valued function (s.v. function for brevity) we define the sets

$$
\begin{aligned}
F(A) & :=\bigcup\{F(x): x \in A\}, \\
F^{-}(B) & :=\{x \in X: F(x) \cap B \neq \emptyset\}, \\
F^{+}(B) & :=\{x \in X: F(x) \subset B\},
\end{aligned}
$$

for every $A \subset X$ and $B \subset Y$.

The composition $G \circ F$ of $F: X \rightarrow n(Y)$ and $G: Y \rightarrow n(Z)$ is the s.v. function given as follows:

$$
(G \circ F)(x):=G(F(x)) \quad \text { for } x \in X .
$$

Assume that $X, Y$ are metric spaces. We say that an s.v. function $F: X \rightarrow n(Y)$ is lower semicontinuous (resp. upper semicontinuous) iff

1991 Mathematics Subject Classification: 54C60, 39B12, 54C65.

Key words and phrases: linear set-valued function, concave set-valued function, iteration semigroup. 
the set $F^{-}(U)$ (resp. $\left.F^{+}(U)\right)$ is open for every open set $U$ in $Y . F$ is said to be continuous iff it is both lower and upper semicontinuous.

A family $\left\{F^{t}: t \geq 0\right\}$ of s.v. functions $F^{t}: X \rightarrow n(X)$ is called an iteration semigroup iff

$$
F^{t} \circ F^{s}=F^{t+s} \quad \text { for all } s, t \geq 0 .
$$

We say that an iteration semigroup $\left\{F^{t}: t \geq 0\right\}$ is continuous iff for every $x \in X$ the s.v. function $t \mapsto F^{t}(x)$ is continuous.

An iteration semigroup $\left\{F^{t}: t \geq 0\right\}$ is concave iff

$$
F^{\lambda s+(1-\lambda) t}(x) \subset \lambda F^{s}(x)+(1-\lambda) F^{t}(x)
$$

for all $s, t \geq 0, \lambda \in[0,1]$ and $x \in X$.

ExAmple 1. The family $\left\{F^{t}: t \geq 0\right\}$ of set-valued functions $F^{t}$ : $[0, \infty) \rightarrow c c([0, \infty))$ given by

$$
F^{t}(x)=e^{t}[0, x], \quad x \in[0, \infty),
$$

is a concave iteration semigroup of linear continuous s.v. functions.

EXAmple 2. Let $G:[0, \infty)^{2} \rightarrow c c\left([0, \infty)^{2}\right)$ be the s.v. function given by $G((x, y))=[0, x] \times[0, y]$. Then the family $\left\{F^{t}: t \geq 0\right\}$ of s.v. functions $F^{t}:[0, \infty)^{2} \rightarrow c c\left([0, \infty)^{2}\right)$ defined by

$$
F^{t}((x, y))=e^{t} G((x, y))
$$

is a concave iteration semigroup of linear continuous s.v. functions.

Before we give the next example we present the correct version of Remark 2 of [5].

REMARK 1. Let $X$ be a Banach space, $C \subset X$ be a closed convex cone and let $G: C \rightarrow c(C)$ be a linear s.v. function satisfying

$$
\begin{aligned}
G^{2}(x) & =G(x) \quad \text { for } x \in C, \\
x & \in G(x) \quad \text { for } x \in C .
\end{aligned}
$$

Then the family $\left\{F^{t}: t \geq 0\right\}$ of s.v. functions

$$
F^{t}(x):=\sum_{i=0}^{\infty} \frac{t^{i}}{i !} G^{i}(x)
$$

is an iteration semigroup of linear continuous s.v. functions.

Proof. Since $G$ satisfies (1), we have

$$
\begin{aligned}
F^{t}(x) & =\sum_{i=0}^{\infty} \frac{t^{i}}{i !} G^{i}(x)=x+\frac{t}{1 !} G(x)+\frac{t^{2}}{2 !} G^{2}(x)+\ldots \\
& =x+\left(\sum_{i=1}^{\infty} \frac{t^{i}}{i !}\right) G(x)=x+\left(e^{t}-1\right) G(x),
\end{aligned}
$$


for all $t \geq 0$ and $x \in C$. Thus the s.v. functions $F^{t}(t \geq 0)$ are linear and continuous with values in $C$. Moreover, by the Theorem of [5], for all $t, s \geq 0$ and $x \in C$ we have

$$
\left(F^{t} \circ F^{s}\right)(x) \subset F^{t+s}(x) .
$$

On the other hand, if $y \in F^{t+s}(x)$, then there exists $z \in G(x)$ such that $y=x+\left(e^{t+s}-1\right) z$. Therefore

$$
\begin{aligned}
y & =x+\left[\left(e^{t}-1\right)\left(e^{s}-1\right)+\left(e^{s}-1\right)+\left(e^{t}-1\right)\right] z \\
& =\left[x+\left(e^{t}-1\right) z\right]+\left(e^{s}-1\right)\left[z+\left(e^{t}-1\right) z\right] .
\end{aligned}
$$

By (2) we can write

$$
\begin{aligned}
y & \in\left[x+\left(e^{t}-1\right) G(x)\right]+\left(e^{s}-1\right)\left[z+\left(e^{t}-1\right) G(z)\right] \\
& \subset\left[x+\left(e^{t}-1\right) G(x)\right]+\left(e^{s}-1\right) \bigcup_{z \in G(x)} F^{t}(z) \\
& =F^{t}(x)+\left(e^{s}-1\right) F^{t}(G(x))=\left(F^{t} \circ F^{s}\right)(x) .
\end{aligned}
$$

Finally $\left(F^{t} \circ F^{s}\right)(x)=F^{t+s}(x)$.

EXAmple 3. Let $G:[0, \infty)^{2} \rightarrow c c\left([0, \infty)^{2}\right)$ be the s.v. function given by $G((x, y))=[0, x] \times[0, y]$. Consider the s.v. functions $F^{t}:[0, \infty)^{2} \rightarrow$ $c c\left([0, \infty)^{2}\right), t \geq 0$, given by

$$
F^{t}((x, y))=\sum_{i=0}^{\infty} \frac{t^{i}}{i !} G^{i}((x, y)) .
$$

Then $\left\{F^{t}: t \geq 0\right\}$ is a concave iteration semigroup of linear continuous s.v. functions of the form

$$
F^{t}((x, y))=(x, y)+\left(e^{t}-1\right) G((x, y)), \quad(x, y) \in[0, \infty)^{2}, t \geq 0 .
$$

Lemma 1 (Lemma 3 of [7]). Let $C$ be a closed convex cone with nonempty interior in a real Banach space $X$ and let $Y$ be a normed space. If $\left\{A_{n}: n \in \mathbb{N}\right\}$ is a sequence of continuous additive s.v. functions $A_{n}: C \rightarrow$ $c c(Y)$ such that $A_{n+1}(x) \subset A_{n}(x)$ for $x \in C$ and $n \in \mathbb{N}$, then the formula

$$
A(x):=\bigcap_{n=1}^{\infty} A_{n}(x)
$$

defines a continuous additive s.v. function $A: C \rightarrow c c(Y)$. Moreover, the sequence $\left\{A_{n}: n \in \mathbb{N}\right\}$ is uniformly convergent to $A$ on every compact subset of $C$.

From now on, Id denotes the set-valued identity, that is, the s.v. function $x \mapsto\{x\}$.

Theorem 1. Assume that $C$ is a closed convex cone with nonempty interior in a Banach space $X$. Let $\left\{F^{t}: t \geq 0\right\}$ be a concave iteration 
semigroup of linear continuous s.v. functions $F^{t}: C \rightarrow c(C)$ such that $F^{0}=$ Id. Then there exists an s.v. function $G: C \rightarrow c c(C)$ such that the family of s.v. functions $\left\{\frac{1}{t}\left(F^{t}-\mathrm{Id}\right): t>0\right\}$ uniformly converges to $G$ on every compact subset of $C$. Moreover, $G$ is linear and continuous and

$$
G(x)=\bigcap_{t>0} \frac{F^{t}(x)-x}{t} \quad \text { for every } x \in C .
$$

Proof. Observe that for all $t, s \geq 0,0 \leq t<s$ and for every $x \in C$,

$$
F^{t}(x)=F^{\frac{t}{s} s+\left(1-\frac{t}{s}\right) 0}(x) \subset \frac{t}{s} F^{s}(x)+\left(1-\frac{t}{s}\right) F^{0}(x) .
$$

Hence

$$
F^{t}(x) \subset \frac{t}{s} F^{s}(x)+\left(1-\frac{t}{s}\right) x, \quad 0 \leq t<s, x \in C,
$$

and consequently

$$
\frac{F^{t}(x)-x}{t} \subset \frac{F^{s}(x)-x}{s}, \quad 0 \leq t<s, x \in C .
$$

This means that $\left\{\frac{1}{t}\left(F^{t}(x)-x\right): t>0\right\}$ is an increasing family of sets, for every $x \in C$. Therefore, by Lemma 1, the s.v. function $G$ given by (3) is linear, continuous and takes nonempty compact convex values in the space $X$. Moreover, for every $x \in C$,

$$
G(x)=\lim _{t \rightarrow 0} \frac{F^{t}(x)-x}{t}
$$

and the convergence is uniform on each compact subset of $C$.

Let $x \in C$. By (3), we have

$$
G(x) \subset \frac{F^{n}(x)-x}{n} \subset C-\frac{1}{n} x
$$

for every positive integer $n$. This implies that $G(x) \subset C$. Therefore $G(x) \in$ $\operatorname{cc}(C)$ for every $x \in C$.

TheOREM 2. Suppose that $C$ is a closed convex cone with nonempty interior in a separable Banach space $X$. Let $\left\{F^{t}: t \geq 0\right\}$ be a concave iteration semigroup of linear continuous s.v. functions $\bar{F}^{t}: C \rightarrow c(C)$ such that $F^{0}=\mathrm{Id}$. Then there exists a linear continuous s.v. function $G: C \rightarrow$ $c c(C)$ such that for every linear continuous selection $g$ of $G$ each of the functions

$$
f^{t}(x):=\sum_{i=0}^{\infty} \frac{t^{i}}{i !} g^{i}(x), \quad x \in C,
$$

is a linear continuous selection of $F^{t}$ for $t \geq 0$ and the family $\left\{f^{t}: t \geq 0\right\}$ is an iteration semigroup. 
Proof. Let $G: C \rightarrow c c(C)$ be given by (3). Then, by Theorem $1, G$ is linear and continuous. Since for every $x \in C$ and $t>0$,

we have

$$
G(x) \subset \frac{F^{t}(x)-x}{t}
$$

$$
x+t G(x) \subset F^{t}(x) \quad \text { for } x \in C, t>0 .
$$

Let $\mathcal{F}_{G}$ be the family of all linear continuous selections of $G$. Then $\mathcal{F}_{G} \neq \emptyset$. Indeed, the Corollary in [6] shows that there exists a linear continuous selection $\widehat{a}$ of $\widehat{G}:=\left.G\right|_{\operatorname{Int} C}$. Let $a$ be the linear continuous extension of $\widehat{a}$ onto the closed cone $C$. Then $a$ is a linear continuous selection of $G$ and consequently $a \in \mathcal{F}_{G}$.

Now, fix any $g \in \mathcal{F}_{G}$. By the Theorem of [5], we can define functions $f^{t}: C \rightarrow C$, for $t>0$, as follows:

$$
f^{t}(x):=\sum_{i=0}^{\infty} \frac{t^{i}}{i !} g^{i}(x), \quad x \in C .
$$

For each $t>0$, we also define

$$
h_{t}(x):=x+\operatorname{tg}(x) \in x+t G(x), \quad x \in C,
$$

which is a linear continuous selection of $F^{t}$ (see (5)).

Fix $t>0$. Observe that for every $x \in C$ we have

$$
h_{t}^{2}(x)=h_{t}\left(h_{t}(x)\right)=x+2 t g(x)+t^{2} g^{2}(x) \in F^{2 t}(x) .
$$

By induction one can prove that for $n \in \mathbb{N}$,

$$
h_{t}^{n}(x)=\sum_{i=0}^{n} \frac{n !}{i !(n-i) !} t^{i} g^{i}(x) \in F^{n t}(x), \quad x \in C .
$$

Set $f_{n}^{t}:=h_{t / n}^{n}$. Then, by the above,

$$
f_{n}^{t}(x)=\sum_{i=0}^{n} \frac{n !}{i !(n-i) !} \frac{t^{i}}{n^{i}} g^{i}(x) \in F^{t}(x), \quad x \in C .
$$

Since for all $n \in \mathbb{N}$ and $i \in\{2, \ldots, n\}$,

$$
\frac{n !}{i !(n-i) !} \cdot \frac{1}{n^{i}}=\frac{1}{i !}\left(1-\frac{i-1}{n}\right) \ldots\left(1-\frac{1}{n}\right)
$$

we can rewrite (7) as follows:

$f_{n}^{t}(x)=x+t g(x)+\sum_{i=2}^{n} \frac{t^{i}}{i !}\left(1-\frac{i-1}{n}\right) \ldots\left(1-\frac{1}{n}\right) g^{i}(x) \in F^{t}(x), \quad x \in C$.

In this way we get a sequence $\left\{f_{n}^{t}: n \in \mathbb{N}\right\}$ of linear continuous selections of $F^{t}$. 
We now show that this sequence converges to the function (6). Let $x \in C$ and let $\varepsilon>0$. Since the series $\sum_{i=0}^{\infty} \frac{(t\|g\|)^{i}}{i !}\|x\|$ is convergent there exists $n_{0} \in \mathbb{N}$ such that

$$
\sum_{i=n}^{\infty} \frac{(t\|g\|)^{i}}{i !}\|x\|<\frac{\varepsilon}{2} \quad \text { for } n>n_{0}
$$

Define

$$
a_{n}^{i}:=\left(1-\frac{i-1}{n}\right) \ldots\left(1-\frac{1}{n}\right)
$$

for $n \in \mathbb{N}, n \geq 2$ and $i \in\{2, \ldots, n\}$. It is easily seen that $0<a_{n}^{i}<1$ $(n \geq 2, i \in\{2, \ldots, n\})$. Moreover for every $i \geq 2$ the sequence $\left\{a_{n}^{i}\right\}_{n \geq i}$ converges to 1 . Therefore there exists $n_{1} \in \mathbb{N}$ such that for all $n>n_{1}$ and $i \in\left\{1, \ldots, n_{0}\right\}$,

$$
\frac{(t\|g\|)^{i}}{i !}\|x\|\left(1-a_{n}^{i}\right)<\frac{\varepsilon}{2\left(n_{0}-1\right)} .
$$

Let $\left\{S_{n}(x): n \in \mathbb{N}\right\}$ be the sequence of partial sums of the series (6). Take any $n>\max \left\{n_{0}, n_{1}\right\}$. Then (8) and (9) yield

$$
\begin{aligned}
\| S_{n}(x) & -f_{n}^{t}(x) \| \\
= & \left\|\sum_{i=0}^{n} \frac{t^{i}}{i !} g^{i}(x)-\left[x+t g(x)+\sum_{i=2}^{n} \frac{t^{i}}{i !}\left(1-\frac{i-1}{n}\right) \cdots\left(1-\frac{1}{n}\right) g^{i}(x)\right]\right\| \\
= & \left\|\sum_{i=2}^{n} \frac{t^{i}}{i !} g^{i}(x)\left(1-a_{n}^{i}\right)\right\| \leq \sum_{i=2}^{n} \frac{(t\|g\|)^{i}}{i !}\|x\|\left(1-a_{n}^{i}\right) \\
= & \sum_{i=2}^{n_{0}} \frac{(t\|g\|)^{i}}{i !}\|x\|\left(1-a_{n}^{i}\right)+\sum_{i=n_{0}+1}^{n} \frac{(t\|g\|)^{i}}{i !}\|x\|\left(1-a_{n}^{i}\right) \\
< & \sum_{i=2}^{n_{0}} \frac{(t\|g\|)^{i}}{i !}\|x\|\left(1-a_{n}^{i}\right)+\sum_{i=n_{0}+1}^{n} \frac{(t\|g\|)^{i}}{i !}\|x\| \\
< & \sum_{i=2}^{n_{0}} \frac{\varepsilon}{2\left(n_{0}-1\right)}+\frac{\varepsilon}{2}=\varepsilon .
\end{aligned}
$$

Hence, since $(7)$ is satisfied and $F^{t}(x) \in c c(C)$ we conclude that $f^{t}(x) \in$ $F^{t}(x)$. Moreover the family $\left\{f^{t}: t \geq 0\right\}$ is an iteration semigroup of linear continuous functions (see Theorem of [5]).

The next theorem is a consequence of Theorem 1 and the Theorem of [4].

Theorem 3. Let $X$ be a separable Banach space, and $C \subset X$ a closed convex cone with nonempty interior. If $\left\{F^{t}: t \geq 0\right\}$ is a concave iteration 
semigroup of linear continuous s.v. functions $F^{t}: C \rightarrow c(C)$ such that $F^{0}=\mathrm{Id}$, then there exists a linear continuous s.v. function $G: C \rightarrow c c(C)$ such that

$$
F^{t}(x) \subset \sum_{i=0}^{\infty} \frac{t^{i}}{i !} G^{i}(x)
$$

for all $t \geq 0$ and $x \in \operatorname{Int} C$.

Proof. By Theorem 1, there exists $G: C \rightarrow c c(C)$ such that

$$
\lim _{t \rightarrow 0} \frac{1}{t}\left(F^{t}(x)-x\right)=G(x), \quad x \in C .
$$

Moreover, the convergence is uniform on every compact subset of $C$. Therefore the semigroup $\left\{F^{t}: t \geq 0\right\}$ satisfies assumptions (i) and (iii) of the Theorem of [4].

Fix $t>0$. Take $n_{0} \in \mathbb{N}$ with $t \leq n_{0}$. By $(3)$,

$$
F^{t}(x)-x \subset t \frac{F^{n}(x)-x}{n} \subset C-\frac{t}{n} x
$$

for all $n \geq n_{0}$ and $x \in C$. This implies that $F^{t}(x)-x \subset C$ for every $x \in C$. Hence condition (ii) of the Theorem of [4] is also satisfied.

Now we show that the semigroup $\left\{F^{t}: t \geq 0\right\}$ is continuous. Fix $x \in C$. By (10), there exists $T>0$ such that for every $0<t \leq T$,

$$
\frac{1}{t}\left(F^{t}(x)-x\right) \subset G(x)+S,
$$

where $S$ is the closed unit ball in $X$. Thus

$$
F^{t}(x)-x \subset t G(x)+t S, \quad 0 \leq t \leq T,
$$

and consequently

$$
\left\|F^{t}(x)-x\right\| \leq T(\|G(x)\|+1)=: m, \quad t \in[0, T] .
$$

Therefore for all $t \in[0, T]$,

$$
F^{t}(x) \subset x+m S .
$$

The above considerations imply that the concave s.v. function $t \mapsto F^{t}(x)$ is bounded on the interval $[0, T]$, and finally it is continuous (see Theorem 4.4 of [3]). Since the semigroup $\left\{F^{t}: t \geq 0\right\}$ satisfies all the assumptions of the Theorem of [4] we have

$$
F^{t}(x) \subset B^{t}(x):=\sum_{i=0}^{\infty} \frac{t^{i}}{i !} G^{i}(x)
$$

for all $t \geq 0$ and $x \in \operatorname{Int} C$. 


\section{References}

[1] C. Castaing and M. Valadier, Convex Analysis and Measurable Multifunctions, Lecture Notes in Math. 580, Springer, Berlin, 1977.

[2] M. Kisielewicz, Differential Inclusions and Optimal Control, PWN, Warszawa, and Kluwer, Dordrecht, 1991.

[3] K. Nikodem, K-convex and K-concave set-valued functions, Zeszyty Nauk. Politech. Łódz. Mat. 559, Rozprawy Nauk. 114, Łódź, 1989

[4] J. Olko, Semigroups of set-valued functions, Publ. Math. Debrecen 51 (1997), 81-96.

[5] J. Plewnia, On a family of set valued functions, Publ. Math. Debrecen 46 (1995), 149-159.

[6] A. Smajdor, Additive selections of a composition of additive set-valued functions, in: Iteration Theory (Batschuns, 1992), World Sci., 1996, 251-254.

[7] - Increasing iteration semigroups of Jensen set-valued functions, Aequationes Math. 56 (1998), 131-142.

Institute of Mathematics

Pedagogical University

Podchorążych 2

30-084 Kraków, Poland

E-mail: olko@polsl.gliwice.pl

Reçu par la Rédaction le 12.2.1998

Révisé le 7.10.1998 